\title{
WILEY-VCH
}

DOI: 10.1002/ ((please add manuscript number))

Article type: Full Paper

\section{D-Printed High Dielectric Contrast Gradient Index Flat Lens for a Directive Antenna with Reduced Dimensions}

Dmitry Isakov ${ }^{1}$, Chris J. Stevens ${ }^{2}$, Flynn Castles $^{1}$ and Patrick S. Grant ${ }^{1}$

1 Department of Materials, University of Oxford, Parks Road, Oxford OX1 3PH, UK.

2 Department of Engineering Science, University of Oxford, Parks Road, Oxford OX1 3PJ, UK

E-mail: dmitry.isakov@materials.ox.ac.uk

Keywords: 3D printing, high-permittivity composite, microwave antenna, gradient refractive index lens

Gradient refractive index (GRIN) materials are of interest for various applications where transformation optic principles can be applied to the design of improved photonic and microwave devices. GRIN materials comprise spatially varying electric and/or magnetic properties that challenge conventional manufacturing processes. In this work we present the design, fabrication, characterisation and performance measurement of a three-dimensional (3D) printed GRIN lens. Using the fused deposition modelling (FDM) 3D printing process with a bespoke filament material possessing high dielectric permittivity a refractive index contrast of $\Delta n=1.4$ across a GRIN lens at Ku-band microwave frequencies is achieved. When the GRIN lens is combined with an open aperture horn, an improved antenna directivity is achieved while simultaneously reducing the overall antenna physical size by over a factor of two.

\section{Introduction}

Transformation optics (TO) is now a well-established design approach for the development of new and advanced electromagnetic devices. TO defines how the spatial pattern of permittivity and permeability in the device can be used to control electromagnetic wave propagation. ${ }^{[1,2]}$ As a result, TO approaches have been theorized and in some cases shown to give rise to a number of desirable functionalities, such as cloaking, ${ }^{[3-7]}$ a field concentrator ${ }^{[9,10]}$ and a 


\section{WILEY-VCH}

photon capturing probe. ${ }^{[11]}$ Additionally, TO approaches can provide innovative alternatives to classic devices, including beam collimators and benders, ${ }^{[12-14]}$ spatially variant photonic crystals and anisotropic materials, ${ }^{[15,16]}$ waveguides $^{[17-19]}$ and power splitters. ${ }^{[20,21]}$

Significant emphasis has been given to the application of TO principles for the development and fabrication of GRIN lenses and directive antennas in the radio frequency range. ${ }^{[22-31]}$ In these examples the beam manipulation mechanism is based on the phase shift of the electromagnetic wave resulting from its interaction with a medium with a spatially graded refractive index. In earlier work, split ring resonators have been used as sub-wavelength unit cells to build a metamaterial-based GRIN medium. ${ }^{[13,28-30]}$ However, these types of metamaterial lenses with wide spatial variation in the refractive index have limited application because of their narrow bandwidth, high dissipation, and the relative difficulty and high cost of fabrication.

Alternatively, spatial variation in refractive index may also be achieved using an "alldielectric" approach in which the GRIN structure is realised by, foe example, patterning air voids within the volume of a polymer lens. ${ }^{[31-36]}$ While this approach has some benefits in ease of fabrication, especially when using $3 \mathrm{D}$ printing technology, ${ }^{[34,37]}$ the range of achievable refractive indices is limited to the range between the dielectric properties of the polymer matrix (with dielectric permittivity $\varepsilon$ in the range of 2 to 2.7) and air ( $\varepsilon=1$ ). To expand this range, appropriately designed voids in different 3D-shaped GRIN lenses were filled with liquid acetonitrile/benzene mixture with a dielectric permittivity of up to $\varepsilon=37$. $^{[38-}$ ${ }^{40]}$ However, the performance of the device is constrained by the relatively high intrinsic dielectric loss of acetonitrile. ${ }^{[41]}$ Alternatively, vacuum casting of titanate powders dispersed in a liquid polymer has been used to fabricate a planar hyperbolic lens. ${ }^{[42]}$ The process involved the manufacture in sequence of concentric layers of the polymer/titanate composite with hemispherical geometry, each with a different permittivity tailored by a change in the local volume fraction of the titanate powder. 


\section{WILEY-VCH}

Despite these advances in the fabrication of objects with relatively complex geometry and broader range of spatially varying refractive index, these processes are comparatively expensive and inflexible. The ability to fabricate new feedstock materials with much higher dielectric permittivity suitable for mass-market fused deposition 3D printing technology has been demonstrated. ${ }^{[43-45]} 3 \mathrm{D}$ printing potentially offers a more flexible and scalable capability for the fabrication objects with complex geometries as well as significant contrast in the spatial variation of refractive index. Using a simple process to mix homogeneously polymer pellets and high permittivity and low-loss powder ceramic titanates into long lengths of composite feedstock filament with a permittivity up to 10, one-, two-, and three-dimensional periodic and graded structures ${ }^{[44]}$ and anisotropic structures with metamaterial features at $12-$ $20 \mathrm{GHz}$ frequency region have been successfully 3D-printed. ${ }^{[45]}$ In this paper we report the design, rapid fabrication using high-dielectric material, and performance of a 3D-printed all-dielectric GRIN lens. The lens was designed to operate in 12-18 GHz frequency range and to facilitate the physical size reduction of a standard antenna horn to a more compact design, without loss of gain or directivity. The standard and compact horn, and the GRIN lens, were all produced by 3D printing. The use of a high permittivity polymer-based composite, alongside a polymer only filament in a "two colour” printing process allowed a progressive increase in the local refractive index contrast within the lens, from the edge to centre. A finite-element based simulation and design suggested a high refractive index contrast GRIN lens could provide a physical size reduction of a horn antenna of up to 3 times. Subsequent manufacture by 3D printing and performance testing gave excellent agreement with simulation predictions, and the compact horn and GRIN lens device showed no significant loss in directivity when compared with an optimised horn geometry operating at the same frequency.

\section{Design of the horn antenna and GRIN lens}




\section{WILEY-VCH}

An electromagnetic wave passing through a boundary between two homogeneous media will experience a phase delay proportional to the refractive index of the media. In a classical lens with a homogeneous index of refraction, the phase shift is generated by the specific profile of the lens surface. Alternatively, a flat lens may replicate the wave phase delay by varying the refractive index along the radius of the lens in all directions. To find the analytical expression for the distribution profile of the refractive index in flat GRIN lens, we consider an arbitrary beam entering a gradient index, planar slab of thickness $t$, emanating from a point source located a distance $f$ from the lens, as shown schematically in Figure 1(a). The exit beam should have the same phase velocity delay as one passing through the center of the lens [46]:

$\frac{f}{\lambda}+\frac{n_{0} t}{\lambda}=\frac{\sqrt{x^{2}+f^{2}}}{\lambda}+\frac{n(x) t}{\lambda}$

where $n_{0}$ and $n(x)$ are index of refraction at the centre of the lens and at a radial distance $x$ respectively, and $\lambda$ is wavelength. From Equation (1), the distribution profile of the refractive index for GRIN lens is given by:

$n(x)=n_{0}-\frac{\sqrt{x^{2}+f^{2}}-f}{t}$

Figure 1(b) shows the spatial distribution of dielectric permittivity $\varepsilon(x)$ for a non- magnetic $(\boldsymbol{n}=\sqrt{\boldsymbol{\varepsilon} \boldsymbol{\mu}}, \mu=1)$ GRIN lens with aperture $A=100 \mathrm{~mm}$ for different thicknesses $t$. Equation (2) shows that an increase in the contrast in refractive index distribution across the lens $(\Delta n=$ $\left.n_{0}-n_{A}\right)$ allows for a decrease in the lens thickness. This feature is used in the present work for the design of an antenna system (comprising a shortened horn and the gradient index lens) with reduced overall length but the same efficiency as a standard optimum horn design with no lens.

A horn antenna is a simple and widely used microwave antenna, used for example as a feed element in large radio astronomy reflectors, satellite tracking and communication systems. The widespread application stems from its simplicity in construction, ease of excitation, versatility and large gain. An H-plane sectoral horn antenna (shown in Figure 2(a)) was 


\section{WILEY-VCH}

chosen in this work due to its simplicity and overall high performance. The optimal directivity of such a horn is obtained when the geometric relation between the horn aperture $A$ and length $R$ is $\boldsymbol{A}=\sqrt{\mathbf{3} \lambda \boldsymbol{R}}$, where $\lambda$ is operating wavelength. ${ }^{[4]}$ Taking this into account, firstly the horn with $R_{r e f}=6 \lambda$ and $\boldsymbol{A}=\lambda \mathbf{3} \sqrt{\mathbf{2}}$ was chosen as the reference antenna for an operating frequency of $15 \mathrm{GHz}$. Secondly, a shortened horn with $R_{\text {short }}=2 \lambda$ and the same aperture was chosen as a compact antenna (Figure 2(b)), and finally the compact horn with GRIN lens was designed (Figure 2(c)). The shortened horn with the GRIN lens has combined length of $60 \mathrm{~mm}$ which is half that of the optimum size reference horn.

The performance of the horns was evaluated using the commercial finite-element analysis package COMSOL Multiphysics. Two-dimensional simulations with the RF Module were conducted to predict and extract the far-field radiation patterns. The same type of simulations was also conducted on the lens itself as part of its design optimisation since the continuous radial variation in refractive index in Equation (2) and performance in Figure 1(b) cannot be 3D-printed readily in practice. Instead the continuous variation must be discretised into subregions of constant permittivity and coarse scale grading achieved by varying the relative thickness of these sub-domains (see later). The parameters of the discretisation of the GRIN lens were retrieved using MATLAB and used for full-wave simulations.

Figure 2 shows full-wave simulation of the electric field propagation radiated at $15 \mathrm{GHz}$ by the reference $R_{r e f}=6 \lambda$ horn (Figure 2(a)), short horn $R_{\text {short }}=2 \lambda$ (Figure 2(b)) and short horn coupled with GRIN lens of thickness $20 \mathrm{~mm}$ with a permittivity profile from Equation (2) (Figure 2(c)). All three antennas have the same aperture size $A=84 \mathrm{~mm}$ and a height $b=7.89$ mm. Reducing the horn size from the optimum strongly undermines the directivity of the horn. However and as intended, the addition of the GRIN lens refocuses the outgoing wave efficiently. These effects are quantified subsequently. 


\section{WILEY-VCH}

\section{Discretization model}

In order to fabricate the gradient refractive index lens, the radial distribution of permittivity (Figure 1(b)) had to be discretized to make it suitable for the dual- filament 3D printer. The design approach consisted of a step-wise approximation of the continuous profile using a discrete number $N$ of composite elements. The principle is presented in Figure 3(a). Using this approximation, the width of the elements $x_{N}$ in the GRIN lens is given by $x_{N}=A / N$, where $A$ is the lens aperture. Each element of the lens is then composed of sub-elements of two materials with relatively low $\varepsilon$ and high $\varepsilon_{\mathrm{h}}$ dielectric permittivity corresponding to ABS only and $\mathrm{ABS} / \mathrm{SrTiO}_{3}$ respectively. The effective local permittivity $\varepsilon_{N}$ of each lens element can be approximated from the relative volume fractions of the low- and high-permittivity subelements according to the upper Wiener bound rule [48]:

$\varepsilon_{N}=f \varepsilon_{h}+(1-f) \varepsilon_{l}$

where $f$ is the fraction of the high permittivity component. To reduce the reflected power and minimize the overall insertion loss due to the lens on the end of the shortened horn, thin input and output impedance matching layers of thickness $3 \mathrm{~mm}$ and $\varepsilon=\varepsilon_{1}$ were also added to the design of the GRIN lens.

The final step in the design of the GRIN lens was the determination of the optimal discrete number $N$ and fraction $f$. Simulations were performed for the directivity of the short horn with the GRIN lens for different values of $N$ and $f$, using real, printed values of $\varepsilon_{1}$ and $\varepsilon_{\text {h }}$. Figure 4(a) shows the simulation results of the directivity retrieved from simulation, where directivity $D$ was defined as the ratio of the peak value of radiated intensity $U(\theta, \varphi)_{\max }$ to the total power radiated from the horn $P(\theta, \varphi)$ over all directions [47]:

$$
D=\frac{U_{\max }}{P / 4 \pi}=\frac{\left.4 \pi U(\theta, \varphi)\right|_{\max }}{\int_{0}^{2 \pi} \int_{0}^{\pi} U(\theta, \varphi) \sin \theta d \theta d \varphi}
$$

where $\theta$ and $\varphi$ are the spherical coordinate angles, and $f(\Theta, \varphi=\pi / 2)$ is an H-plane cut. The reference horn (optimised size, no lens) and the short horn with an "ideal” continuous radial 


\section{WILEY-VCH}

varying GRIN lens are also shown: the “ideal” lens improves directivity significantly. For the practical, discretised lens the directivity is improved for all $N$ in the range $10-25$ with little additional benefit beyond $N=20$. Figure 4(b) shows similar simulations at $N=20$ but a range of $\varepsilon=7$ to 10 . Interestingly, because an increase in $\varepsilon_{\mathrm{h}}$ reduces the fraction of high permittivity material needed in each sub-element, the lens becomes more inhomogeneous at the coarser scale and performance is degraded. After further detailed optimisation, the optimal parameters for the lens were $N=21$ and $\varepsilon_{\mathrm{h}}=7$.

\section{Experimental}

The horns and the GRIN lens were printed using a dual-extrusion Makerbot Replicator 2 desktop 3D printer with standard temperature and layer resolution settings using $0.4 \mathrm{~mm}$ diameter print nozzles. To print regions with either relatively low or high permittivity, two types of feedstock filament were used: commercially supplied acrylonitrile butadiene styrene (ABS) was used for the low dielectric permittivity regions $\left(\varepsilon_{1}=2.60, \tan \delta=4.80 \times 10^{-3}\right.$ at 15 $\mathrm{GHz}$ ), while high dielectric permittivity regions used a composite filament comprising 28 vol. $\% \mathrm{SrTiO}_{3}\left(<3 \mu \mathrm{m}\right.$, Sigma-Aldrich) powder in an ABS matrix $\left(\varepsilon_{\mathrm{h}}=7.20, \tan \delta=7.66 \times\right.$ $10^{-3}$ at $15 \mathrm{GHz}$ ). Details of the high-permittivity filament fabrication and their detailed permittivity characterisation can be found in Refs. [44,45].

The dielectric properties of the low and high-permittivity filaments were measured from 3Dprinted coupons using a $15 \mathrm{GHz}$ split-post dielectric resonator (QWED, Warsaw) ${ }^{[49]}$ and a Rohde \& Schwarz ZNB20 vector network analyser.

The performance of the 3D-printed reference horn and the short horn+GRIN lens system was measured from angle-dependent far-field TE polarization radiation patterns in the xy plane. The experiment was conducted in an anechoic chamber using the 3D- printed transmission and a 3D-printed receiver horn. The as-printed horns were copper coated (Caswell, copper conductive ink, $79 \mu \Omega \mathrm{cm}$ vol. resistivity). Measurements of the radiation patterns were 


\section{WILEY-VCH}

performed in the 12-18 GHz frequency range using an Agilent 5071C vector network analyser with a transmission horn mounted on synchronized turntable.

The spatial variation of the dielectric permittivity of the lens itself was characterized using a single split ring resonator probe moving in an $x y$-plane above the top face of the lens with $0.20 \mathrm{~mm}$ step resolution. The probe comprised a split ring (formed from a $5 \mathrm{~mm}$ section of 22 mm diameter copper tube of wall thickness $0.8 \mathrm{~mm}$ ) fixed between near field coupled transmitting and receiving ports connected to a VNA. The resulting resonant frequency of this split ring arrangement depended on the ring geometry and capacity of the air gap in the region of the split in the ring. If a material with dielectric permittivity greater than air is placed close $(<0.5 \mathrm{~mm})$ to the gap in the resonator ring, the ring resonant frequency shifts lower as a monotonic function of dielectric constant being probed and therefore the method can be used as a spatially sensitive surface dielectric probe.

\section{Performance and Discussion}

Figure 5(a) shows a photograph of the 3D-printed standard optimum H-plane horn antenna and shortened horn with GRIN lens. In accordance with the design, the GRIN lens was printed using two dielectric materials for each of the sub-elements. The GRIN lens took approximately $2.5 \mathrm{~h}$ to print as a single component in one step, direct from the optimized CAD file. Figure 5(b) shows a close-up image of the 3D-printed lens and experimental measurements of the spatial distribution of the dielectric permittivity measured by a single split ring resonator probe moving over the surface of the lens in discrete steps. The local effective permittivity of the 3D-printed GRIN lens was in reasonable agreement with the optimised continuous variation from Equation (2).

Figure 6 presents the experimental far-field radiation pattern in the azimuthal plane for all three antenna systems: the reference $\left(R_{r e f}=6 \lambda\right)$ horn, short $\left(R_{\text {short }}=2 \lambda\right)$ horn, and the short horn coupled with the printed GRIN lens. Both, the reference horn and the short horn with the 


\section{WILEY-VCH}

GRIN lens were significantly more directive. The sidelobes of both these antennas were below $-35 \mathrm{~dB}$ and the half-power beam width was in the range $10-14^{\circ}$. The small deviation of approximately $3 \%$ of the axis of the beam from $0^{\circ}$ direction for the short horn with the GRIN lens (Figure 6(d)) was caused by the slight asymmetry in the distribution of the dielectric permittivity in the 3D-printed GRIN lens, as shown on close inspection of Figure 5(b). Figure 7 shows the measured directivity retrieved from the radiation pattern for all three antenna systems. The data confirms the trend shown in the simulations in Figure 4 whereby shortening the horn to a third of the length destroyed its directivity. As also suggested by simulations at the design stage in Figure 4, adding the GRIN lens, which increased overall length but to only half of the standard horn, recovered directivity to that of the reference horn, to within experimental accuracy.

\section{Conclusions}

A consideration of bespoke materials development, model guided design and 3D printing have been used to produce an optimised and discretised graded index lens for microwave applications. Standard modelling approaches were sufficiently sensitive and flexible to represent the discretized GRIN lens performance readily, and predictions were in good agreement with experiment using microwaves centred at $15 \mathrm{GHz}$. Critically, models were constrained by the range of permittivities that could be easily and reproducibly realised in a 3D-printed lens. Once an optimum design was selected, it was quickly fabricated by 3D printing using bespoke composite filament with an as-printed permittivity of up to 7.2. The 3D-printed GRIN lens allowed a reduction in size of a horn antenna system of 50\%, with no loss of directivity performance. Although a relatively simple device, this experimental demonstration suggests considerable opportunity for 3D printing to enable radical TOinspired devices in the microwave domain, especially as higher dielectric constant materials suitable for 3D printing become available. 


\section{WILEY-VCH}

\section{Acknowledgements}

This work was funded by the UK Engineering and Physical Sciences Research Council $(\mathrm{EP} / \mathrm{I034548/1)}$

Received: ((will be filled in by the editorial staff))

Revised: ((will be filled in by the editorial staff)) Published online: ((will be filled in by the editorial staff))

[1] U. Leonhardt, Science 2006 312, 1777.

[2] J. B. Pendry, D. Schurig, D. R. Smith, Science 2006 312, 1780.

[3] N. B. Kundtz, D. R. Smith, J. B. Pendry, Proc. IEEE 2011 99, 1622.

[4] D. P. Gaillot, C. Cronne, F. Zhang, D. Lippens, New J. Phys. 2008 10, 115039.

[5] J. Valentine, J. Li, T. Zentgraf, G. Bartal, X. Zhang, Nat. Mater. 2009 8, 568.

[6] T. Ergin, N. Stenger, P. Brenner, J. B. Pendry, M. Wegener, Science 2010 328, 337.

[7] D. Diedrich, A. Rottler, D. Heitmann, S. Mendach. New J. Phys. 2012 14, 053042.

[8] D. M. Nguyen, H. Xu, Y. Zhang, B. Zhang, Appl. Phys. Lett. 2015 107, 121901.

[9] M. M. Sadeghi, S. Li, L. Xu, B. Hou, H. Chen, Sci. Rep. 2015 5, 8680.

[10] M. Rahm, D. Schurig, D. A. Roberts, S. A. Cummer, D. R. Smith, J. B. Pendry, Photon. Nanostr. 2008 6. 87.

[11] J. B. Pendry, A. I. Fernndez-Domnguez, Y. Luo, R. Zhao, Nat. Phys. 2013 9, 518522.

[12] D. Kwon, D. H. Werner, New J. Phys. 2008 10, 115023.

[13] Z. L. Mei, T. J. Cui, Opt. Express 2009 17, 18354.

[14] M. Rahm, D. A. Roberts, J. B. Pendry, D. R. Smith, Opt. Express 2008 16, 11555.

[15] R. C. Rumpf, J. J. Pazos, J. Opt. Soc. Am. A 2013 30, 1297.

[16] R. C. Rumpf, J. J. Pazos J L Digaum, S. M. Kuebler, Philos. Trans. R. Soc. A 2015 373, 20140359.

[17] P. H. Tichit, S. N. Burokur, A. Lustrac, J. Appl. Phys. 2014 115, 024901.

[18] H. Y. Xu, H. D. Sun, B. L. Zhang, Sci. China. Inf. Sci. 2013 56, 120403.

[19] D. Shin, J. Kim, D.-S. Yoo, K. Kim, Opt. Express 2015 23, 21892. 


\section{WILEY-VCH}

[20] W. Shu, S. Yang, W. Yan, Y. Ke, T. Smith, Opt. Commun. 2015 338, 307.

[21] G. Gok, A. Grbic, Phys. Rev. Lett. 2013 111, 233904.

[22] H. F. Ma, X. Chen, X. M. Yang, W. X. Jiang, T. J. Cui, J. Appl. Phys. 2010107 014902.

[23] Z. Lei Mei, J. Bai, T. J. Cui, J. Phys. D: Appl. Phys. 2010 43, 055404.

[24] X. Chen, H. F. Ma, X. Y. Zou, W. X. Jiang, T. J. Cui, J. Appl. Phys. 2011 110, 044904.

[25] P. H. Tichit, S. N. Burokur, D. Germain, A. Lustrac, Phys. Rev. B 2011 83, 155108.

[26] I. Aghanejad, H. Abiri, A. Yahaghi, IEEE Trans. Antennas Propagat. 2012 60, 4074.

[27] B. Q. Lu, Z. H. Jiang, D. H. Werner, Antennas Wirel. Propag. Lett. 2015 13, 1779.

[28] D. R. Smith, J. J. Mock, A. F. Starr, D. Schurig, Phys. Rev. E 2005 71, 036609.

[29] R. Liu, Q. Cheng, J. Y. Chin, J. J. Mock, T. J. Cui, D. R. Smith, Opt. Express 2009 17, 21030.

[30] H. F. Ma, X. Chen, H. S. Xu, X. M. Yang, W. X. Jiang, T. J. Cui Appl. Phys. Lett. 2009 95, 094107.

[31] H. F. Ma, B. G. Cai, T. X. Zhang, Y. Yang, W. X. Jiang, T. J. Cui, IEEE Trans. Antennas Propagat. 2013 61, 2561.

[32] X. Zhou, X. Zou, Y. Yang, H. Ma, T. J. Cui, Sci. China Inf. Sci. 2013 56, 120410.

[33] M. Q. Qi, W. X. Tang, T. J. Cui, Sci.Rep. 2015511732.

[34] R. C. Rumpf, C. R. Garcia, H. H. Tsang, J. E. Padilla, M. D. Irwin, Prog. Electromagn. Res. 2013 142, 243.

[35] F. Y. Meng, R. Z. Liu, K. Zhang, D. Erni, Q. Wu, L. Sun, Prog. Electromagn. Res. $2013141,17$.

[36] H. F. Ma, T. J. Cui, Nature Commun. 2010 1, 124.

[37] J. Yl, S. N. Burokur, G.-P. Piau, A. Lustrac, Sci. Rep. 2015 6, 18819.

[38] M. Yin, T. X. Yong, W. L. Ling, L. D. Chen, Appl. Phys. Lett. 2014 104, 094101. 


\section{WILEY-VCH}

[39] H. Han, L. Wu, X. Tian, D. Li, M. Yin, Y. Wang, J. Appl. Phys. 2012 112, 114913.

[40] L. Wu, X. Tian, M. Yin, D. Li, Y. Tang, Appl. Phys. Lett. 2013 103, 084102.

[41] J. Barthel, M. Kleebauer, R. Buchner, J. Sol. Chem. 1995 24, 1.

[42] O. Quevedo-Teruel, W. Tang, R. C. Mitchell-Thomas, A. Dyke, H. Dyke, L. Zhang, S. Haq, Y. Hao, Sci. Rep. 2013 3, 1903.

[43] P. S. Grant, F. Castles, Q. Lei, Y. Wang, J. M. Janurudin, D. Isakov, S. Speller, C.

Dancer, C. R. M. Grovenor, Philos. Trans. R. Soc. A 2015 373, 20140353.

[44] F. Castles, D. Isakov, A. Lui, Q. Lei, C. Dancer, Y. Wang, J. M. Janurudin, D. Speller, C. R. M. Grovenor, P. S. Grant, Sci. Rep. 2016 6, 22714.

[45] D. V. Isakov, Q. Lei, F. Castles, C. J .Stevens, C. R. M. Grovenor, P. S. Grant, Mater. Des. 2016 93, 423.

[46] D. Meschede, Optics, Light and Lasers: The Practical Approach to Modern Aspects of Photonics and Laser Physics, 2nd Ed. John Wiley \& Sons 2008.

[47] C. A. Balanis, Antenna Theory: Analysis and Design, 3rd Ed. John Wiley \& Sons 2005.

[48] A. Sihvola, Subsurf. Sens. Technol. Appl. 2000 1, 393.

[49] J. Krupka, A. P. Gregory, O. C. Rochard, R. N. Clarke, J. Eur. Ceram. Soc. 2001 21, 2673.

(a)

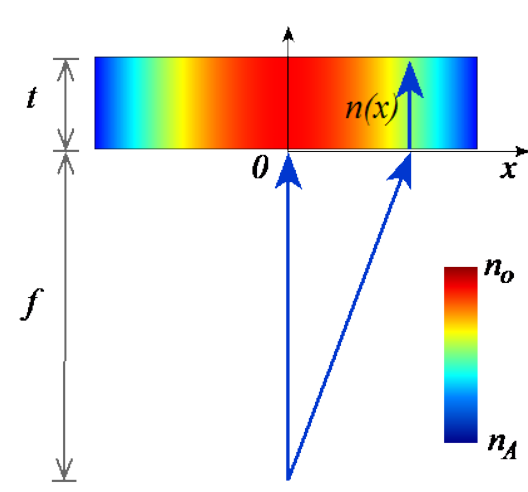

(b)

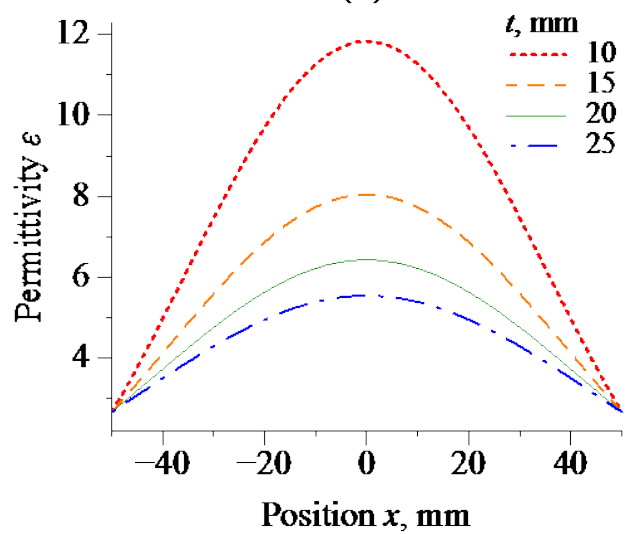

Figure 1. (a) Schematic diagram of the GRIN lens; and (b) the distribution of the dielectric permittivity for different thicknesses of the GRIN lens from Equation (2). 


\section{WILEY-VCH}

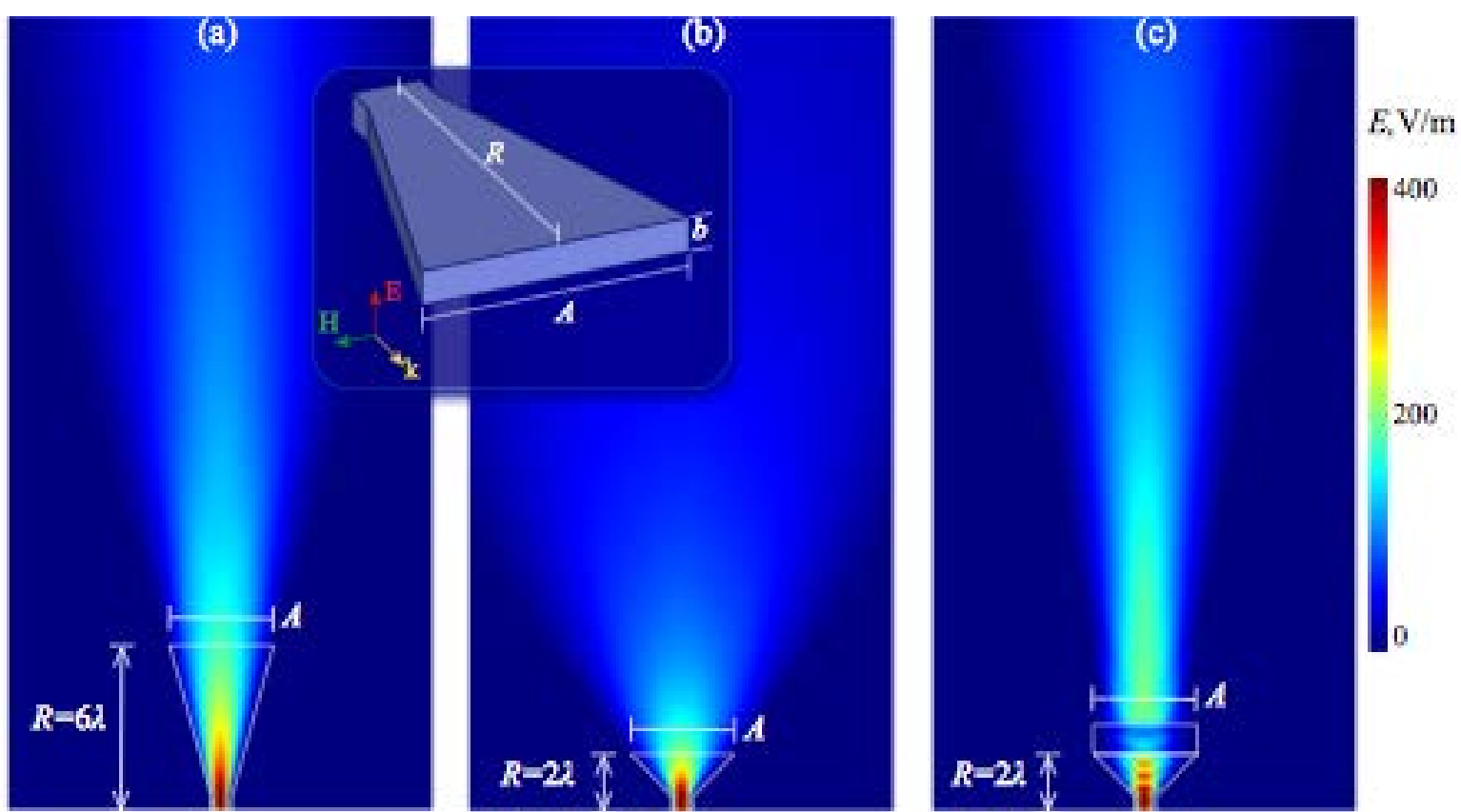

Figure 2. (a) Simulated electric field distribution in proposed antenna systems: (a) reference $\left(R_{\text {ref }}=6 \lambda\right)$; (b) short $\left(R_{\text {short }}=2 \lambda\right)$ horn; and (c) short horn coupled with GRIN lens. The colour bar is uniform for all three patterns. Inset shows an H-plane horn.

(a)

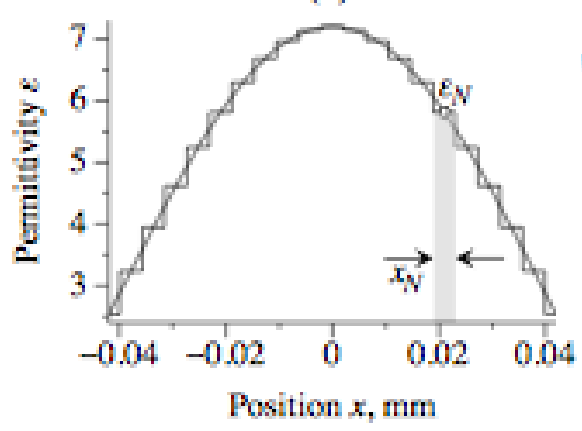

(b)

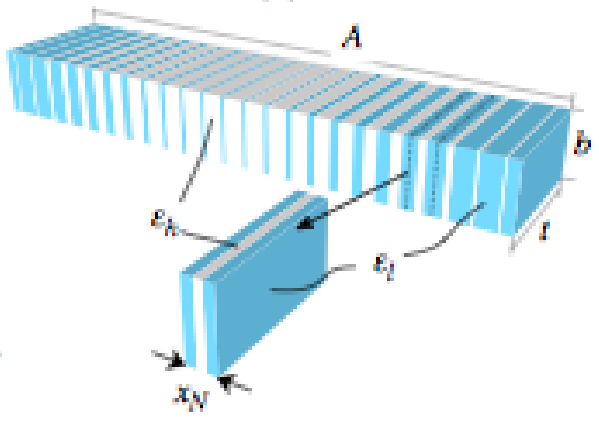

Figure 3. (a) Continuous and discrete distribution of dielectric permittivity in the GRIN lens with thickness $t=20 \mathrm{~mm}$ and aperture $A=84 \mathrm{~mm}$; (b) corresponding CAD model of the GRIN lens with discrete distribution of permittivity obtained from alternating, varying width sub-elements based relatively low- and high-permittivity regions.
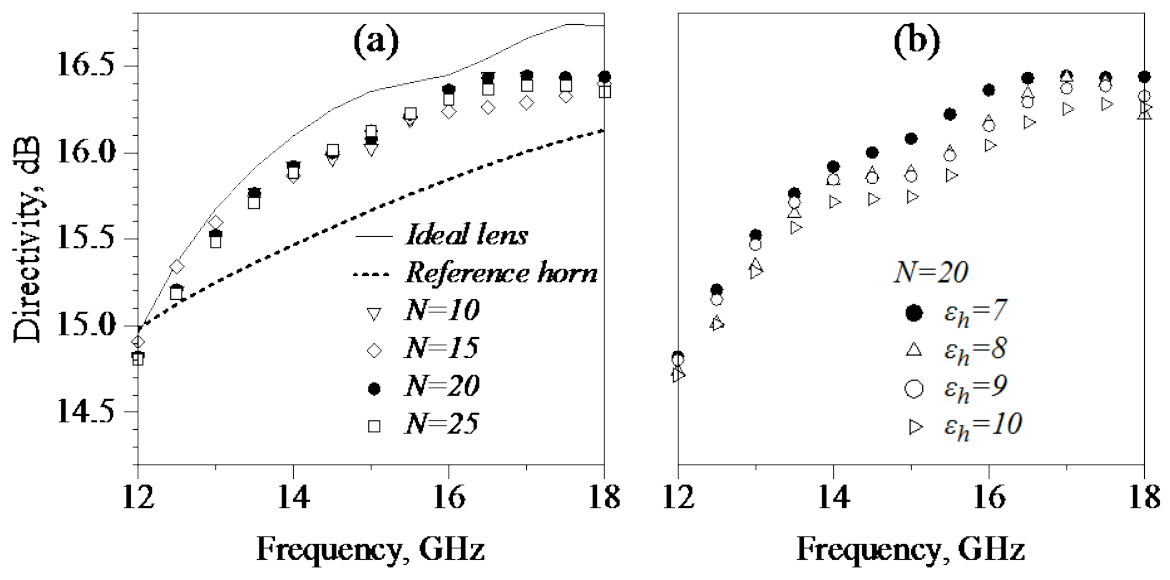


\section{WILEY-VCH}

Figure 4. Simulated directivity of (a) the reference horn, the shortened horn with an ideal continuous variation GRIN lens, and the shortened horn with a discretised GRIN lens of $N$ elements; and (b) the shortened horn with GRIN lens with $N=20$ elements and sub-elements with high permittivity regions in the range $\varepsilon_{\mathrm{h}}=7$ to 10 .
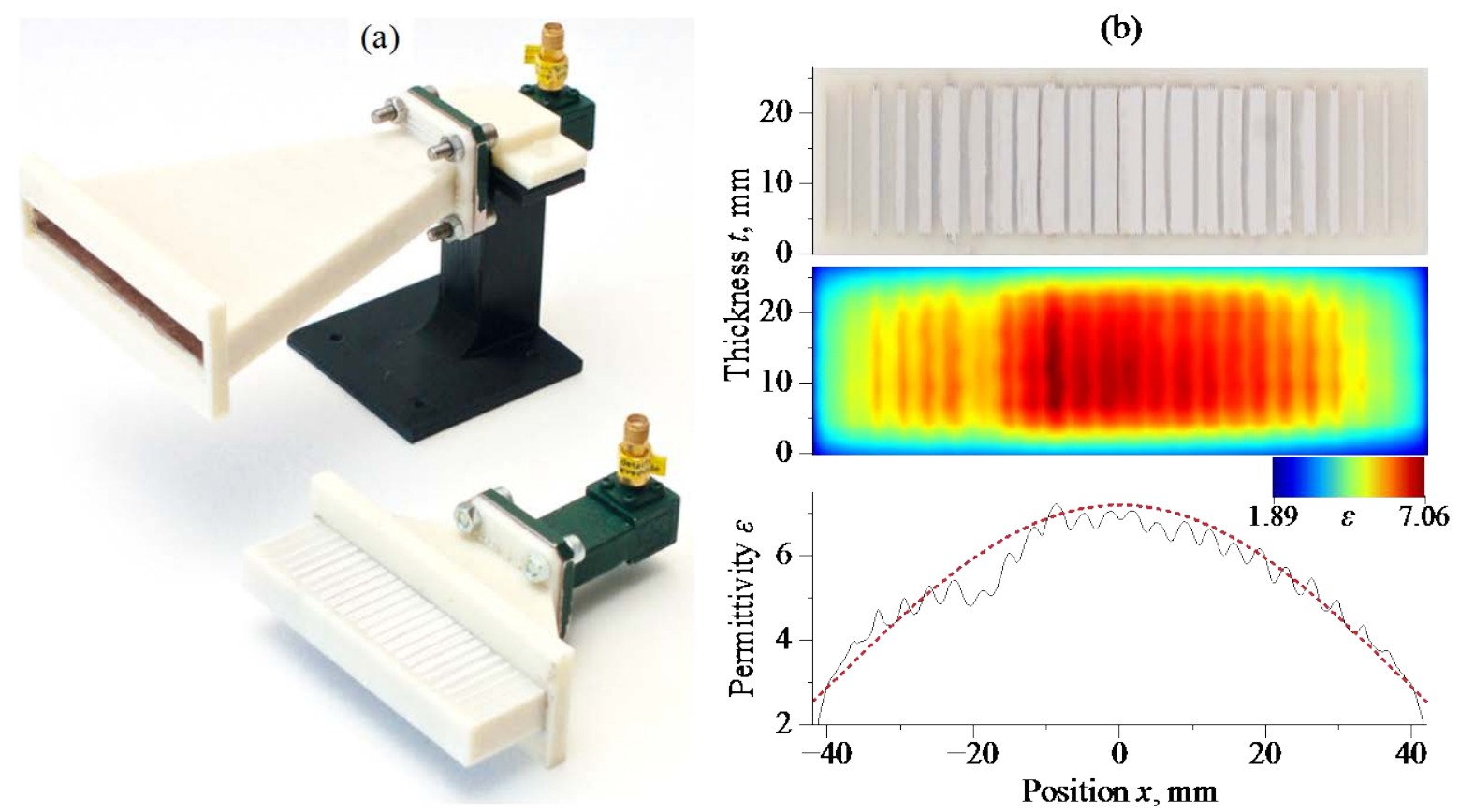

Figure 5. (a) Photograph of the 3D-printed antennas and gradient index lens; (b) close-up of the 3D-printed lens, experimental measurements of the spatial distribution of dielectric permittivity over the lens surface using a split ring resonator and a comparison of the measured (solid line) and ideal theoretical (dotted line) radial variation in local permittivity. 


\section{WILEY-VCH}

(a)

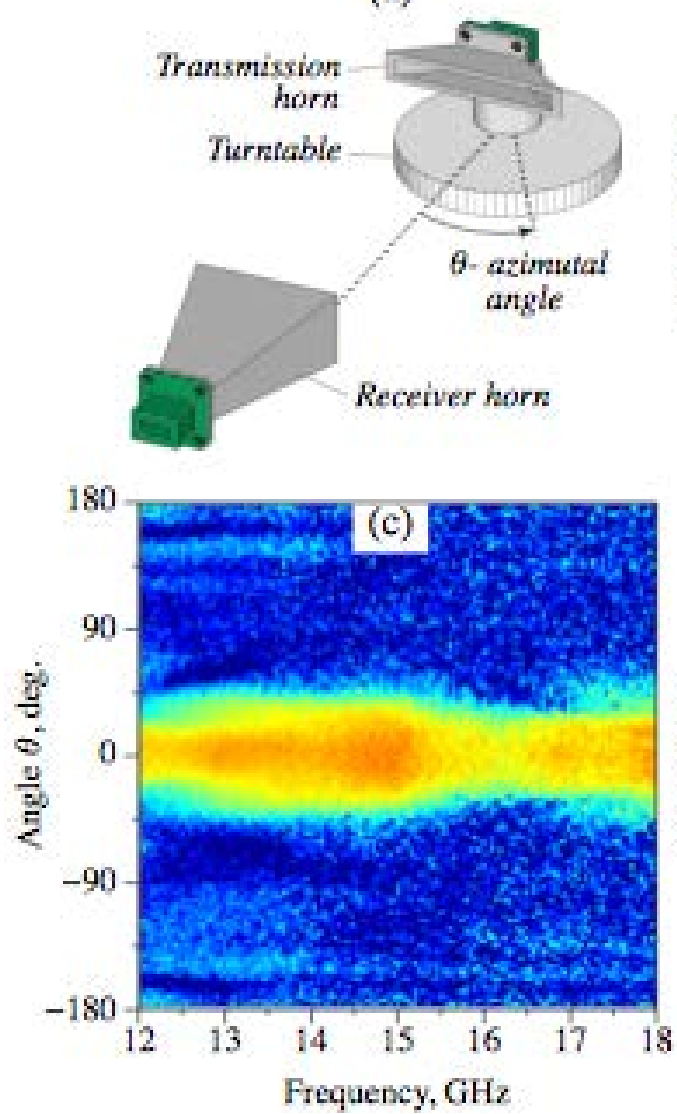

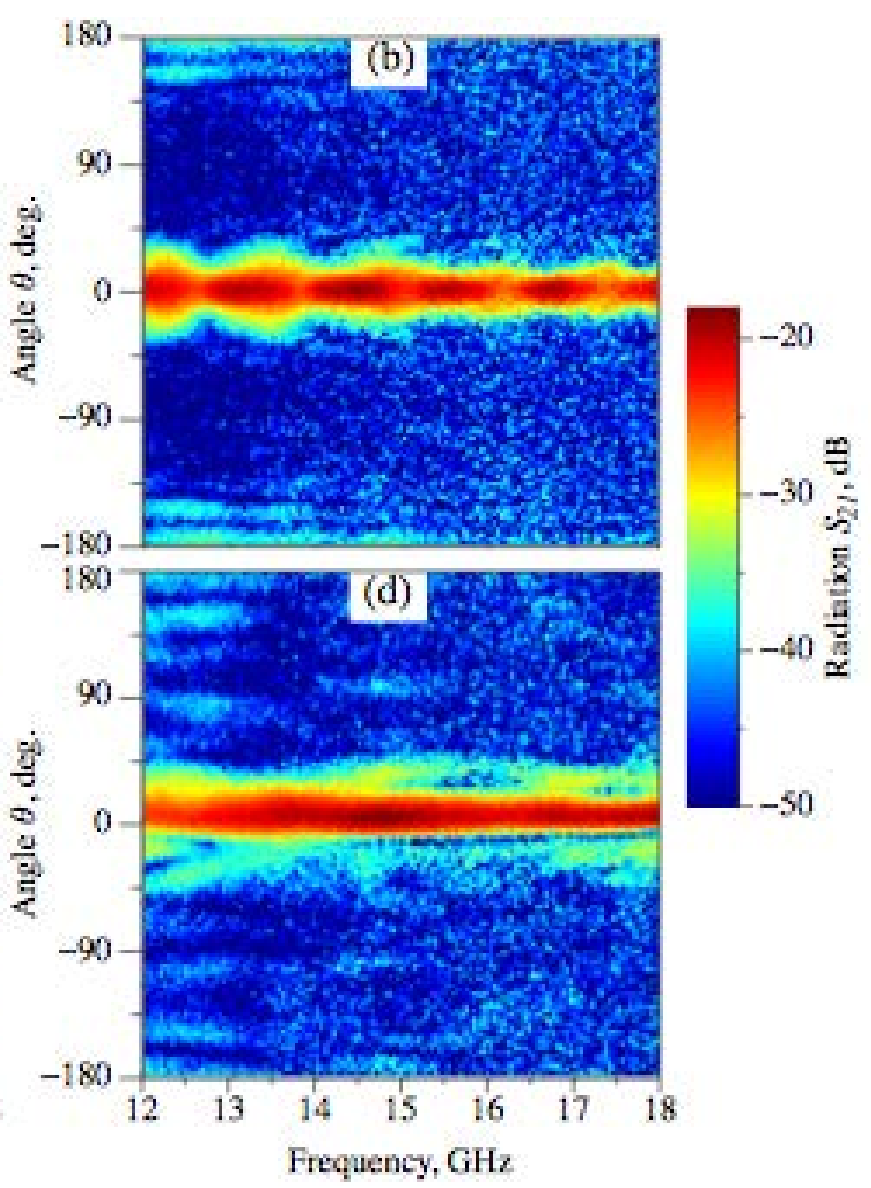

Figure 6. (a) Schematic arrangement of the experiment; (b) radiation patterns (from $S_{21}$ data) of the optimised reference horn, (c) radiation pattern of the short horn, and (d) radiation pattern of the short horn with 3D-printed GRIN lens.

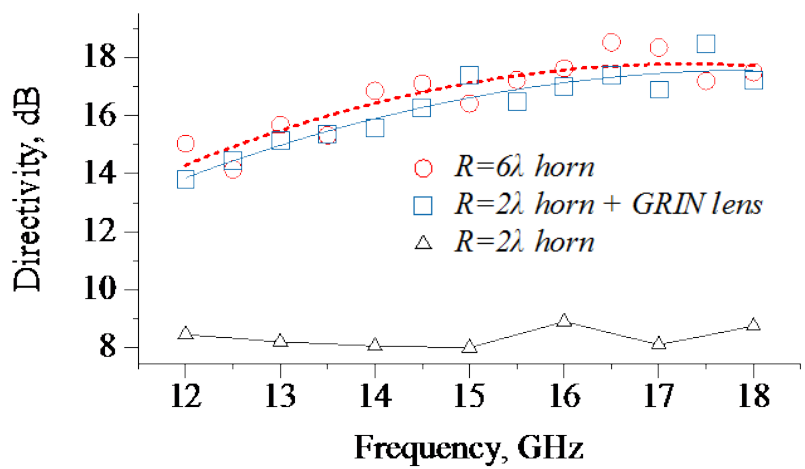

Figure 7. The directivity of the 3D-printed antennas as a function of frequency. 


\section{WILEY-VCH}

Rapid fabrication and performance of a 3D-printed all-dielectric gradient index (GRIN) flat lens is presented. The use of a bespoke high permittivity polymer-based composite in 3D printing process allows a progressive increase in the local refractive index contrast within the lens. 3D-printed GRIN lens device shows no significant loss in directivity when compared with an optimised horn geometry operating at the same frequency.

Keyword 3D printing, high-permittivity composite, gradient refractive index lens

D. Isakov*, C. J. Stevens, F. Castles, P. S. Grant

Title 3D-Printed High Dielectric Contrast Gradient Index Flat Lens for a Directive Antenna with Reduced Dimensions
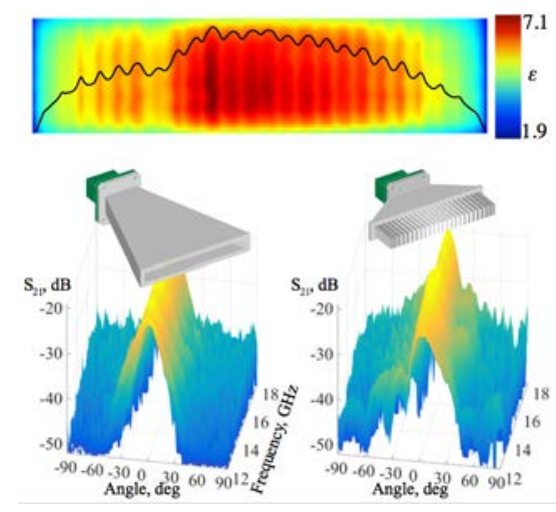
WILEY-VCH 